\title{
Relative Energy Deficiency in Sport among a Collegiate Cross-Country Team*
}

\author{
Regina L. Schimek ${ }^{1}$, Elizabeth Blodgett Salafia ${ }^{2}$, Sherri Stastny ${ }^{{ }^{*}}$ \\ ${ }^{1}$ Department of Health, Nutrition, and Exercise Sciences, North Dakota State University, Fargo, North Dakota \\ ${ }^{2}$ Department of Human Development and Family Science, North Dakota State University, Fargo, North Dakota \\ Email: regina.schimek@ndsu.edu, elizabeth.blodgettsalafia@ndsu.edu, ^sherri.stastny@ndsu.edu
}

How to cite this paper: Schimek, R.L., Salafia, E.B. and Stastny, S. (2021) Relative Energy Deficiency in Sport among a Collegiate Cross-Country Team. Open Journal of Internal Medicine, 11, 81-90. https://doi.org/10.4236/ojim.2021.112007

Received: March 31, 2021

Accepted: May 22, 2021

Published: May 25, 2021

Copyright (C) 2021 by author(s) and Scientific Research Publishing Inc. This work is licensed under the Creative Commons Attribution International License (CC BY 4.0).

http://creativecommons.org/licenses/by/4.0/ (c) (i) Open Access

\begin{abstract}
Objective: To investigate risk for relative energy deficiency in sports (RED-S) among a team of female and male collegiate long-distance runners. Methods: North Dakota State University (Fargo, ND, USA) female and male competitive long-distance runners who agreed to participate during fall semester, 2019, completed an electronic survey containing the LEAF-Q and EAT-26 surveys and additionally completed a DXA scan, to screen for low energy availability and RED-S, disordered eating/eating disorders, and bone health, respectively. Participants were required to be $18-25$ years old, not injured, training a minimum of five hours per week, and currently participating as part of a collegiate competitive team. Thirteen participants $($ male $=3)$ finished the study which also included energy intake and exercise expenditure self-reported with a three-day food diary and exercise log and estimated with ESHA Food Processor. Response rate was $42 \%$ of entire team; mean age 19.8. Results: The LEAF-Q significantly predicted risk for RED-S ( $p<0.007)$. Correlations using the participants EAT-26 score, energy availability levels ( $\mathrm{p}$ $<0.508)$, and whether they were at risk for RED-S ( $p<0.208$ ) or not were non-significant for all comparisons, although there was a moderate positive correlation between EAT-26 and RED-S risk $(\mathrm{R}=0.454)$. Participants at risk for RED-S had higher occurrences of injuries $(p<0.022)$ and lower DXA measured Z-scores $(p<0.063)$ than those not at risk for RED-S. Conclusion: Long distance runners at risk for RED-S may have higher occurrences of injuries and lower bone mineral density, which can be easily measured using available screeners and DXA technology. What are the new findings: • Long distance runners at risk for RED-S may have higher occurrences of injuries and lower bone mineral density then age- and sex-matched individuals. How might it impact on clinical practice in the near future: • This is important for
\end{abstract}

${ }^{\star}$ Each author contributed to the writing of this manuscript. Data is available upon reasonable request from sherri.stastny@ndsu.edu. 
future clinical practice to be aware of the potential negative impacts on the bone development in young adults; - Particularly long-distance runners or athletes in leanness focused sports should be screened for energy availability which can impact performance, and future bone density.

\section{Keywords}

RED-S, Long-Distance Runners, Bone Mineral Density

\section{Introduction}

The purpose of this study was to investigate relative energy deficiency in sport (RED-S) in collegiate long-distance runners and determine associations with bone health. RED-S, defined as a syndrome that impairs physiological function, and includes but is not limited to impairments of metabolic rate, menstrual function, bone health, immunity, protein synthesis, and cardiovascular health is a condition in which low energy availability (LEA) results in negative effects on health and performance in both females and males [1]. A lack of knowledge regarding RED-S exists among coaches, athletic trainers and athletes [2].

The etiology of RED-S was identified and defined by the International Olympic Committee (IOC) in 2014 to serve as a more comprehensive explanation for all individuals who were experiencing symptoms similar to that of the Female Athlete Triad (Triad) [1]. The Triad is known as a condition influencing three components of female athlete health including energy availability, menstrual function, and bone health [3]. Energy deficiency is the etiological factor at the base of the Triad; therefore, causing the identified physiological symptoms [1]. However, the Triad is not inclusive of males experiencing physiological symptoms of energy deficiency [1]. Therefore, the term RED-S was developed to include the physiological dysfunction that both males and females experience in a state of energy deficiency.

To further understand RED-S and its role in overall athlete well-being, it is critical to describe the basic terms that are used to identify RED-S. Low energy availability is the etiological factor of RED-S that causes physiological impairment. Energy availability is the energy consumed through dietary intake minus the energy expended in exercise [4]. Exercise energy expenditure (EEE) is the expenditure of energy above the normal daily needs of living used during exercise [4]. The calculation is depicted in Equation (1).

$$
\begin{aligned}
& \text { Energy Availability }(\mathrm{EA}) \\
& =[\text { Energy intake }(\mathrm{EI})(\mathrm{kcal})-\text { Exercise Energy Expenditure }] \\
& \qquad(\mathrm{EEE})(\mathrm{kcal}) \div \text { Fat-Free Mass }(\mathrm{FFM})(\mathrm{kg})
\end{aligned}
$$

On average, optimal EA is achieved at 45 kilocalories per kilogram of fat-free mass (FFM) per day (kcal/kg FFM/day) [4]. When EA is less than $30 \mathrm{kcal}$ per $\mathrm{kg}$ 
FFM, negative physiological changes begin to occur. For example, energy use is diverted to essential functions, optimal metabolic function can become disturbed, and physiological functions disabled [5] [6]. Such processes can impact different areas of health and performance such as menstrual function, bone, immunological health, metabolic and hematologic function, growth and development, endocrine, psychological, cardiovascular, and gastrointestinal systems, and have been correlated with depression, irritability, impaired judgement, increased risk for injury, decreased glycogen stores, decreased muscle strength, decreased endurance performance, impaired training response, decreased coordination, and decreased concentration [2]. Athletes who participate in endurance sports, such as running, and non-weight-bearing sports, often have lower bone mineral density (BMD) than athletes participating in other track and field events [7].

Little research regarding energy deficiency, and associations with bone and eating health among long-distance runners inclusive of males at a collegiate level have been reported. The aim of this study was to examine the effectiveness of RED-S screening tools to help identify athletes that may be at risk for current or future compromised bone health.

\section{Methods}

A cross-sectional study included collegiate female and male competitive longdistance runners from North Dakota State University during fall semester, 2019. Participants were required to be 18 - 25 years old, not injured, training a minimum of five hours per week, and currently participating as part of a collegiate competitive team. Athletes meeting study requirements were invited to an informational meeting to learn about the study. The session included a mini-nutritional lesson regarding nutrients needed for bone health, e.g. adequate energy, protein, calcium and vitamin $\mathrm{D}$.

\subsection{Questionnaire}

Each athlete was invited to participate in an electronic survey (Qualtrics, Provo, UT) that combined the EAT-26 survey to screen for eating disorders [8], the LEAF-Q to screen for RED-S [9], and a three-day food diary and exercise log. The EAT-26 consisted of 26 questions, each consisting of a 6-point scale of "never" $=0$ to "always" $=6$ points. A score $\geq$ to 20 points. Participant scores of 20 or greater indicated disordered eating or an eating disorder. The LEAF-Q is a validated 25-item questionnaire assessing physiological symptoms related to energy deficiency such as menstrual dysfunction, history of injury, and gastrointestinal function [9]. Additionally, the study questionnaire included demographic questions, plus select questions to probe for bone-health related occurrences such as, "have you experienced any injuries during the last 12 months that have prevented your participation in training or competition?" Study procedures were clearly presented on the informed consent approved by the univer- 
sity IRB. After required consents and health screening forms were completed, participants completed a DXA scan (Lunar Prodigy, GE Healthcare, Waukesha, WI) to determine bone density and fat-free mass.

To be at risk for RED-S a female participant had to score an 8 or higher on the LEAF-Q. Male participants who scored a 3.2 or higher were considered at risk for RED-S. This was determined by elimination of questions that were not applicable to males. Questions used to determine males at risk for RED-S were based on methods used in a study investigating LEA in athletes [10]. For the purposes of this study it is considered a low energy availability in males' questionnaire (LEAM-Q), a study-specific tool designed for this investigation.

\subsection{Food Diary and Exercise Log}

For the three-day food diary, participants were asked to keep a detailed record of all food and beverage intake for two week days and one weekend day (or two "normal days" and one "abnormal eating" day). To effectively estimate total EI, participant logging was guided by a registered dietitian. Energy intake and exercise expenditure were analyzed using ESHA Food Processor (Salem, OR). Exercise energy expenditure was self-reported using a three-day exercise log. This log included type of exercise, duration, and intensity. Participants were asked to log exercise the same days as the food log. Participants used rate of perceived exertion to determine the intensity of the exercise training. Additionally, a manual heart rate measurement was collected as a low heart rate indicates risk of LEA [11].

\subsection{Participant Report Card}

Participants received results from the DXA scan and the nutritional analysis, including the EAT-26 score, at the end of the study in the form of a "participant report card" (see Figure 1). If a participant scored high for eating disorders on the EAT-26, had problems with bone health, or were energy deficient, they were advised to see their physician for a complete health screening and follow-up.

\subsection{Statistical Analysis}

An independent samples $t$-test was used to compare the BMI of those at risk for RED-S and those not at risk of RED-S as determined by LEAF-Q or LEAM-Q score. A Chi square test for independence was used to determine if female and male runners who screened positive for RED-S have low energy availability. An independent samples $t$-test compared EA levels of those at risk for RED-S and those not at risk for RED-S. Pearson's correlation was used to determine if female and male distance runners who are at risk for disordered eating are also at risk for RED-S and low energy availability. Energy availability for all participants was determined using the EA calculator [12]. Linear regression was used to determine if female and male distance runners who are at risk for disordered eating or who have low energy availability become more susceptible to bone injury. 


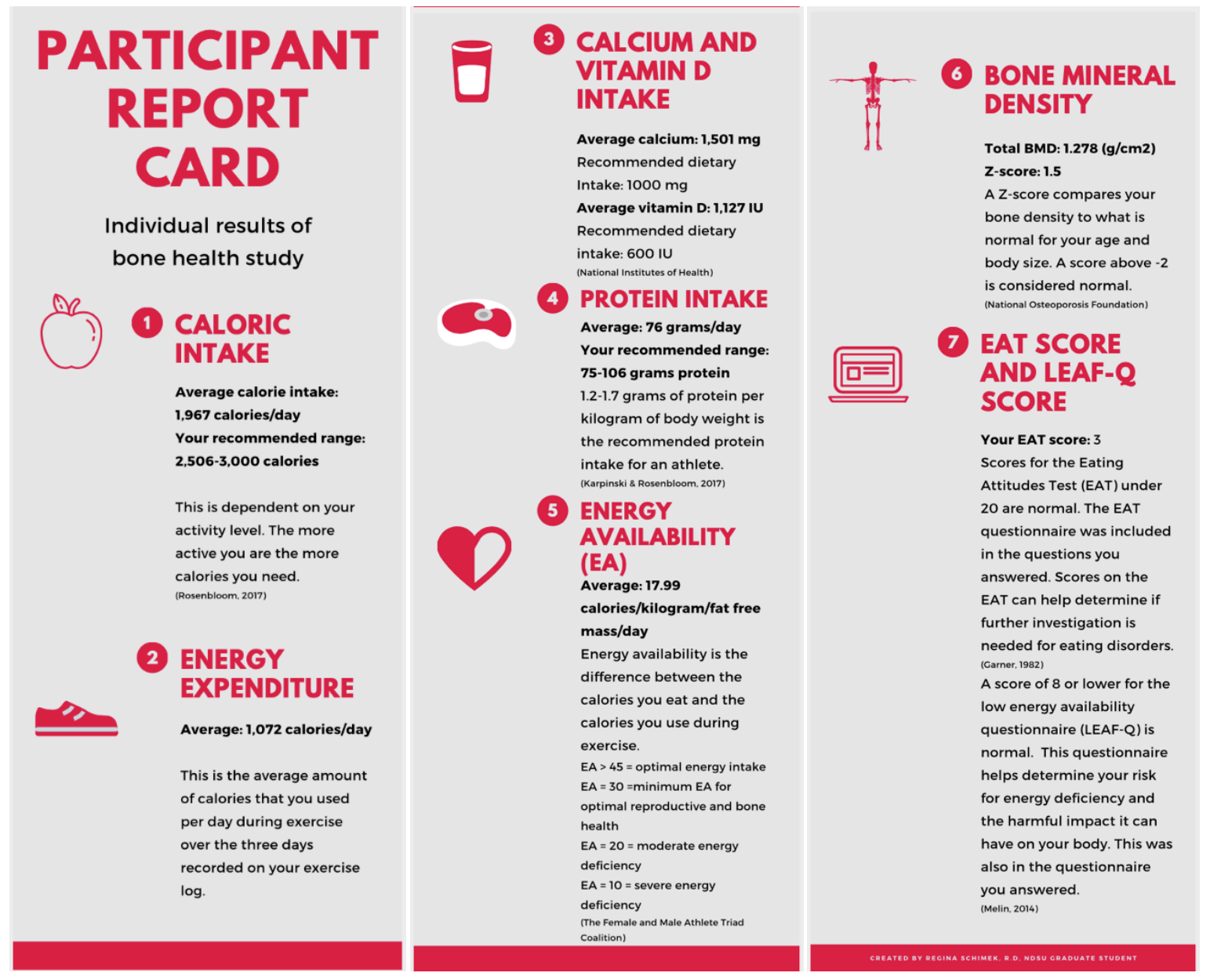

Figure 1. Example of individual participant report card for bone health study among long-distance runners.

\section{Results}

A total of 13 collegiate (male $=3$ ) long-distance runners participated in this study. The percent rate of participation was $42 \%$ out of the potential population, with no drop outs. Results from LEAF-Q, LEAM-Q, and RED-S screens along with comparison of differences and means for all variables are displayed in $\mathrm{Ta}$ ble 1 .

An independent samples $t$-test of all participant EAT-26 scores in comparison to whether they were at risk for RED-S or not was non-significant, though the p-value is skewed due to an outlier score of 45 . Correlations using the participants EAT-26 score, EA levels, and whether they were at risk for RED-S or not were non-significant for all comparisons, although there was a moderate positive correlation between EAT-26 and RED-S risk $(\mathrm{R}=0.454)$.

A linear regression used to determine if the participants EAT-26 score predicted if they had experienced injury in those at risk for RED-S and those not at risk for RED-S was non-significant. When comparing those at risk of RED-S to those not at risk of RED-S with their energy availability levels as a predictor and whether or not they reported experiencing injuries as a dependent variable, 
Table 1. Results from the low energy availability in female's questionnaire (LEAF-Q) to determine participants at risk for RED-S.

\begin{tabular}{|c|c|c|c|c|c|c|}
\hline & $\begin{array}{c}\text { All }(\mathbf{n}=13) \\
\text { Mean age } 19.8\end{array}$ & $\begin{array}{l}\text { Women } \\
(n=10)\end{array}$ & $\begin{array}{l}\text { Men } \\
(n=3)\end{array}$ & $\begin{array}{l}\text { LEAF/M-Q } \\
(\mathrm{n}=7 \text { at risk) }\end{array}$ & $\begin{array}{c}\text { LEAF/M-Q } \\
(\mathrm{n}=6 \text { not at risk) }\end{array}$ & $P$ value \\
\hline BMI & $20.59 \pm 1.05$ & $20.46 \pm 0.85$ & $21.03 \pm 1.11$ & $20.67 \pm 0.60$ & $20.50 \pm 1.49$ & $\mathrm{p}<0.208$ \\
\hline LEAF-Q score & $8.38 \pm 4.82$ & $5.0 \pm 4.90$ & $2.00 \pm 2.08$ & $11.43 \pm 4.57$ & $4.83 \pm 1.60$ & $\mathrm{p}<0.007$ \\
\hline EAT-26 score & $6.53 \pm 11.77$ & $7.40 \pm 3.45$ & $3.60 \pm 0.57$ & $10.42 \pm 15.40$ & $2.00 \pm 1.41$ & $\mathrm{p}<0.079$ \\
\hline EI (kcal) & $2487.38 \pm 642.36$ & $2359.87 \pm 607.84$ & $2912.39 \pm 678.95$ & $2571.16 \pm 651.49$ & $2389.63 \pm 678.01$ & \\
\hline EEE (kcal) & $669.96 \pm 211.93$ & $643.09 \pm 215.95$ & $759.54 \pm 209.74$ & $671.02 \pm 281.40$ & $668.73 \pm 112.99$ & \\
\hline EA (kcal·kg FFM·day) & $32.36 \pm 10.11$ & $31.81 \pm 10.45$ & $34.21 \pm 10.70$ & $34.52 \pm 10.43$ & $29.84 \pm 10.02$ & $\mathrm{p}<0.457$ \\
\hline RMR (kcal/day) & $1359.07 \pm 150.48$ & $1323.10 \pm 93.23$ & $1479.39 \pm 262.12$ & $1322.57 \pm 116$ & $1401.66 \pm 185$ & $\mathrm{p}<0.419$ \\
\hline Resting heart rate (bpm)* & $51.53 \pm 5.89$ & $53.0 \pm 5.90$ & $46.66 \pm 2.30$ & $52.00 \pm 5.65$ & $51.00 \pm 6.66$ & $\mathrm{p}<0.654$ \\
\hline Total BMD $\left(\mathrm{g} / \mathrm{cm}^{2}\right)$ & $1.22 \pm 0.07$ & $1.21 \pm 0.06$ & $1.28 \pm 0.08$ & $1.20 \pm 0.07$ & $1.25 \pm 0.07$ & $\mathrm{p}<0.178$ \\
\hline Z-Score & $0.86 \pm 0.63$ & $0.93 \pm 0.62$ & $0.66 \pm 0.75$ & $0.57 \pm 0.61$ & $1.21 \pm 0.48$ & $\mathrm{p}<0.063$ \\
\hline Injuries & $1.53 \pm 1.85$ & $1.1 \pm 1.66$ & $3.00 \pm 2.00$ & $2.57 \pm 1.98$ & $0.33 \pm 0.51$ & $\mathrm{p}<0.010$ \\
\hline
\end{tabular}

Note: ${ }^{*}$ The generally accepted range for resting heart rate is 60 to 100 beats per minute. The "red flag" range for cardiac abnormality is lower than 50 beats per minute [23].

marginal significance was indicated $(\mathrm{p}<0.080)$. Seven out of thirteen participants reported experiencing some type of injury on the LEAF-Q survey. Injuries reported included plantar fasciitis, tendinitis, overuse of hamstring, quad pull, strain in SI joint, and stress fracture in femur. Mean dietary calcium intake was $1625 \mathrm{mg}$ with a range of $1001 \mathrm{mg}$ to $3054 \mathrm{mg}$.

\section{Discussion}

The aim of this study was to investigate RED-S in female and male collegiate athletes and determine if there is an association with bone health. Key findings included a strong association between risk of RED-S and number of reported injuries. Also, Z-scores of those at risk of RED-S were lower (though only marginally significant). The American College of Sports Medicine (ACSM) defines low BMD as "history of ... nutritional deficiencies, stress fractures ... and a BMD Z-score of -1.0 to -2.0 " [3]. The ISCD recommends $Z$-scores be used to determine BMD in adolescent and premenopausal populations [13]. The mean age for participants in the current study was 19.8 years old. However, when comparing total BMD for those at risk for RED-S and those not at risk for RED-S there was no significant difference and the mean scores were very similar.

\subsection{Injury Reported with LEAF/M-Q}

Participants at risk for RED-S had a higher number of reported injuries. Six out of the seven participants who were at risk for RED-S reported experiencing at least one injury. Only two of the six participants who were not at risk for RED-S reported experiencing at least one injury. An increased risk for injury is one of 
the components in the possible negative performance effects of RED-S [1]. A study by Heikura et al. concluded that athletes with low reproductive hormones (e.g. testosterone in males and amenorrheic females) had 4.5 times higher rate of absences in training due to bone injury [14]. Although the current study did not collect information on hormones, it is well-known that low energy availability causes hormone imbalance in both males and females [1].

Low energy availability can be indicated by a BMI of less than $17.5 \mathrm{~kg} \cdot \mathrm{m}^{-2}$ [15]. There was no significance between participant BMI of those who were at risk for RED-S and those not at risk for RED-S in the current study. This differs from the Tornberg et al. study that was structured to determine if there was a link between athletes with secondary functional hypothalamic amenorrhea and neuromuscular performance [16]. Athletes with secondary functional hypothalamic amenorrhea had lower BMI's compared to athletes with eumenorrhea [16]. Amenorrhea is one of the indicators for RED-S [17].

\subsection{Energy Availability and EAT-26/LEAF-Q Screeners}

Overall, the average EA was $32.36 \mathrm{kcal} \cdot \mathrm{kg} \cdot \mathrm{FFM} \cdot \mathrm{day}^{-1}$. Optimal EA is considered $\geq 45 \mathrm{kcal} \cdot \mathrm{kg} \cdot \mathrm{FFM} \cdot \mathrm{day}^{-1}$; when EA drops below $30 \mathrm{kcal} \cdot \mathrm{kg} \cdot \mathrm{FFM} \cdot \mathrm{day}^{-1}$ or less, negative physiological changes begin to occur [4]. Based on the three-day food diaries, six of the thirteen participants had EA below $30 \mathrm{kcal} \cdot \mathrm{kg} \cdot \mathrm{FFM} \cdot \mathrm{day}^{-1}$. Six participants had estimated EA between 30 and $45 \mathrm{kcal} \cdot \mathrm{kg} \cdot \mathrm{FFM} \cdot \mathrm{day}^{-1}$. For the current study, seven out of thirteen participants were at risk for RED-S by scoring an $\geq 8$ or $\geq 3.2$ on the LEAF-Q and LEAM-Q, respectively. A similar study found that females with amenorrhea and males with lower testosterone had a significant difference in scores on the LEAF-Q and the Triad tool than females with eumenorrhea and males with low compared to moderate EA [14] [18]. Though there was no significance between participants who were at risk for RED-S and LEA $(p<0.429)$, only one participant had what is considered optimal EA of $45 \mathrm{kcal} \cdot \mathrm{kg} \cdot \mathrm{FFM} \cdot \mathrm{day}^{-1}$. Evidence suggests that some individuals may be more sensitive to LEA than others and may be more likely to experience negative physiological symptoms [19]. There was a marginal significance found when comparing EA levels and whether or not they had reported experiencing an injury between those at risk for RED-S and those not at risk for RED-S ( $p<0.080)$. Therefore, EA of those at risk for RED-S was marginally significant when predicting if a participant had experienced an injury. Injuries or bone injuries, and LEA are components that are often paired together.

Scores greater than 20 on the EAT-26 indicate a need for further investigation by a qualified professional [8]. Only one participant scored higher than 20 (score of 45) on the EAT-26. This same participant also had LEA and was at risk for RED-S based on the LEAF-Q score (23.61 EA kcal-kg.FFM.day ${ }^{-1}, 15$ LEAF-Q score). A study investigated whether the presence of dieting behaviors, disordered eating, and level of body dissatisfaction for female athletes was impacted by the level of sport such as elite, recreational, or noncompetitive. The EAT-26 
was used to identify if the participants were at risk for an eating disorder or disordered eating that warranted further investigation by a professional [20]. As a result, more disordered eating patterns were noticed in leanness-important sport and elite athletes. Participants in the current study are in what is considered a leanness focused or weight sensitive sport and are at a collegiate level (i.e. crosscountry competitive running).

There was no significance between runners who were at risk for disordered eating by taking the EAT-26 test and scoring 20 or higher and susceptibility to bone injury. A small sample size may explain why many of the tests, including this comparison, were non-significant. Athletes are potentially at higher risk for eating disorders than non-athletes due to the pressure and focus put on the body for performance, or particularly for sports that have more focus on aesthetic characteristics [21]. In an acute interventional study, Papageorgiou et al. found that five days of LEA, at $15 \mathrm{kcal} \cdot \mathrm{kg} \cdot \mathrm{LBM} \cdot \mathrm{day}^{-1}$, decreased bone formation and increased bone resorption among women [22].

There is an association between negative psychological factors and LEA [9]. The LEAF-Q is meant to compliment disordered eating/eating disorder screening tools. These screening tools can indicate if athletes are at risk for LEA which is often linked to eating disorders and amenorrhea [9]. Scores comparing LEAF-Q and LEAM-Q of those at risk for RED-S and those not at risk for RED-S was significant. Those with RED-S had a higher mean score (11.43) than those not at risk for RED-S (4.83). Seven out of the thirteen participants in this study scored high enough to be considered at risk for RED-S.

\section{Conclusion}

Overall, this study determined that both male and female athletes who are at risk for RED-S have a greater chance of experiencing a higher number of injuries than athletes not at risk for RED-S. It is important to note that over half of the participants were at risk for RED-S. With a larger sample size additional results may be found for the relationship between RED-S and bone health in male and female athletes. Screening is recommended on both male and female athletes to determine risk for RED-S and its impact on overall athlete health and performance in variety of sports.

\section{Conflicts of Interest}

The authors declare no conflicts of interest regarding the publication of this paper.

\section{References}

[1] Mountjoy, M., Sundgot-Borgen, J., Burke, L., et al. (2014) The IOC Consensus Statement: Beyond the Female Athlete Triad-Relative Energy Deficiency in Sport (RED-S). British Journal of Sports Medicine, 48, 491-497. https://doi.org/10.1136/bjsports-2014-093502

[2] Mountjoy, M., Sundgot-Borgen, J., Burke, L., et al. (2018) International Olympic 
Committee (IOC) Consensus Statement on Relative Energy Deficiency in Sport (RED-S): 2018 Update. International Journal of Sport Nutrition and Exercise Metabolism, 28, 316-331. https://doi.org/10.1123/ijsnem.2018-0136

[3] Nattiv, A., Loucks, A.B., Manore, M.M., et al. (2007) The Female Athlete Triad. Medicine \& Science in Sports \& Exercise, 39, 1867-1882. https://doi.org/10.1249/mss.0b013e318149f111

[4] Loucks, A.B., Kiens, B. and Wright, H.H. (2011) Energy Availability in Athletes. Journal of Sports Sciences 29, S7-S15. https://doi.org/10.1080/02640414.2011.588958

[5] Dufour, D.L. and Sauther, M.L. (2002) Comparative and Evolutionary Dimensions of the Energetics of Human Pregnancy and Lactation. American Journal of Human Biology, 14, 584-602. https://doi.org/10.1002/ajhb.10071

[6] Jasienska, G. (2003) Energy Metabolism and the Evolution of Reproductive Suppression in the Human Female. Acta Biotheoretica, 51, 1-18. https://doi.org/10.1023/A:1023035321162

[7] Scofield, K.L. and Hecht, S. (2012) Bone Health in Endurance Athletes: Runners, Cyclists, and Swimmers. Current Sports Medicine Reports, 11, 328-334. https://doi.org/10.1249/JSR.0b013e3182779193

[8] Garner, D.M. and Garfinkel, P.E. (1979) The Eating Attitudes Test: An Index of the Symptoms of Anorexia Nervosa. Psychological Medicine, 9, 273-279. https://doi.org/10.1017/S0033291700030762

[9] Melin, A., Tornberg, A.B., Skouby, S., et al. (2014) The LEAF Questionnaire: A Screening Tool for the Identification of Female Athletes at Risk for the Female Athlete Triad. British Journal of Sports Medicine, 48, 540-545. https://doi.org/10.1136/bjsports-2013-093240

[10] Slater, J. (2015) Low Energy Availability in New Zealand Recreational Athletes. Master Thesis, University of Otago, Dunedin.

[11] O’Donnell, E., Goodman, J.M., Mak, S., et al. (2015) Discordant Orthostatic Reflex Renin-Angiotensin and Sympathoneural Responses in Premenopausal ExercisingHypoestrogenic Women. Hypertension, 65, 1089-1095. https://doi.org/10.1161/HYPERTENSIONAHA.114.04976

[12] A Collaboration of the National Collegiate Athletic Association (NCAA) Sports, Cardiovascular, and Wellness Nutrition (SCAN) \& Collegiate and Professional Sports Dietitians Association (CPSDA).

https://www.sportsrd.org/wp-content/uploads/2018/11/Energy Availability Fact S heet WEB.pdf

[13] Leslie, W.D., Adler, R.A., El-Hajj Fuleihan, G., et al. (2006) Application of the 1994 WHO Classification to Populations Other than Postmenopausal Caucasian Women: The 2005 ISCD Official Positions. Journal of Clinical Densitometry, 9, 22-30. https://doi.org/10.1016/j.jocd.2006.05.004

[14] Heikura, I.A., Uusitalo, A.L.T., Stellingwerff, T., et al. (2018) Low Energy Availability Is Difficult to Assess but Outcomes Have Large Impact on Bone Injury Rates in Elite Distance Athletes. International Journal of Sport Nutrition and Exercise Metabolism, 28, 403-411. https://doi.org/10.1123/ijsnem.2017-0313

[15] Joy, E., De Souza, M.J., Nattiv, A., et al. (2014) 2014 Female Athlete Triad Coalition Consensus Statement on Treatment and Return to Play of the Female Athlete Triad. Current Sports Medicine Reports, 13, 219-232.

[16] Tornberg, Å.B., Melin, A., Koivula, F.M., et al. (2017) Reduced Neuromuscular Performance in Amenorrheic Elite Endurance Athletes. Medicine \& Science in 
Sports \& Exercise, 49, 2478-2485. https://doi.org/10.1249/MSS.0000000000001383

[17] Sygo, J., Coates, A.M., Sesbreno, E., et al. (2018) Prevalence of Indicators of Low Energy Availability in Elite Female Sprinters. International Journal of Sport Nutrition and Exercise Metabolism, 28, 490-496.

https://doi.org/10.1123/ijsnem.2017-0397

[18] Joy, E., De Souza, M.J., Nattiv, A., et al. (2014) 2014 Female Athlete Triad Coalition Consensus Statement on Treatment and Return to Play of the Female Athlete Triad. Current Sports Medicine Reports, 13, 219-232.

https://doi.org/10.1249/JSR.0000000000000077

[19] Cialdella-Kam, L., Guebels, C.P., Maddalozzo, G.F. and Manore, M.M. (2014) Dietary Intervention Restored Menses in Female Athletes with Exercise-Associated Menstrual Dysfunction with Limited Impact on Bone and Muscle Health. Nutrients, 6, 3018-3039. https://doi.org/10.3390/nu6083018

[20] Kong, P. and Harris, L.M. (2015) The Sporting Body: Body Image and Eating Disorder Symptomatology among Female Athletes from Leanness Focused and Nonleanness Focused Sports. The Journal of Psychology, 149, 141-60 https://doi.org/10.1080/00223980.2013.846291

[21] Martinsen, M. and Sundgot-Borgen, J. (2013) Higher Prevalence of Eating Disorders among Adolescent Elite Athletes than Controls. Medicine \& Science in Sports \& Exercise, 45, 1188-1197. https://doi.org/10.1249/MSS.0b013e318281a939

[22] Papageorgiou, M., Elliott-Sale, K.J., Parsons, A., et al. (2017) Effects of Reduced Energy Availability on Bone Metabolism in Women and Men. Bone, 105, 191-199. https://doi.org/10.1016/j.bone.2017.08.019

[23] Karpinski, C. and Rosenbloom, C.A. (2017) Sports Nutrition: A Handbook for Professionals. 6th Edition, Academy of Nutrition and Dietetics, Chicago 